\title{
Research on Numerical Method for Fluid-Structure Interaction in Engine Blades
}

\author{
Zhen Tan ${ }^{1,2, a}$, Peng Zhang ${ }^{1}$, Guangyu Du ${ }^{1}$, Bangchun Wen ${ }^{1}$ \\ ${ }^{1}$ School of Mechanical Engineering and Automation, Northeastern University, Shenyang, 110819 \\ China \\ ${ }^{2}$ Shen Yang Broadcasting And TV University, Shenyang, 110003 China \\ aE-mail: tanz_hen@yahoo.cn
}

Keywords: Fluid-structure interaction, CFD, CSD, Engine blades, Numerical analysis method

\begin{abstract}
A numerical analysis method for fluid-structure interaction (FSI) to analyze engine blades dynamic response was presented. Fluid-structure interaction is an important research field. It is mostly studies the interaction between the influence upon the fluid by the deformation of the solids, the important characteristic of fluid-solid interaction mechanics is the fluid-solid interaction between the both phase mediums. The solutions of strongly coupling and weakly coupling were discussed firstly in this paper. We compared the advantages and disadvantages of the strongly coupling and weakly coupling. And using numerical analysis method based on weakly coupling, we established a fluid-solid interaction control equation taking solid and fluid as a unified mathematical model. And we study about blades deformation and displacement under the action of air loading in engine. Using computational structural dynamics (CSD) calculate the displacements of blades, and using computational fluidic dynamics (CFD) calculate the pressures of blades, completing the fluid-structure interaction analysis in engine blades by iterating this two values(the displacements and the pressures) until the computational convergence solution is obtained. At the end of this paper, the model of fluid-structure interaction and the simulate procession of the numerical analysis method were presented. Based on the analysis, the simulation result is qualitatively discussed referring to the factual conditions of the engine for validating the feasibility of analysis method.
\end{abstract}

\section{Introduction}

Among the many scientific and engineering fields, the objects of study are set out by the various types of physical loads in the structure, such as: aerodynamic loads, thermal loads, electrical loads, etc., in order to get more accurate results, different kind of subjects need to be coupled with, mainly on account of the coupling of two or more the result of the interaction between physical fields. FSI which is an important area of research, the main consideration fluid coupling between the fluid and solid structure interaction, and its main feature is the interaction between the two-phase medium, the deformation of the solid in the fluid loading will occur deformation or movement, solid deformation or movement, in turn, changes the distribution and size of the fluid load.

Fluid-structure interaction problems because of its cross-cutting characteristic, and various engineering fields, such as civil, aerospace, marine, power, marine, petrochemical, mechanical, nuclear, geological and biological engineering have a relationship with it. Particularly important in aerospace applications, the current research more and more attention has been paid in this area, many disciplines of research methods were successfully applied into this study. In this paper, the method for fluid-structure interaction in engine blades was reviewed, and the application of fluid-solid coupling algorithm was proposed [1]. 


\section{Mathematical Method}

The dynamics analysis of fluid-solid coupling has two methods: one is analytical -- numerical method, which uses finite element discrete of the structure and uses approximate analytic description of the fluid; the other which is pure numerical methods such as finite element method, finite difference method, boundary element method and mixed.

At present, because the actual project strongly nonlinear problems, analytical method to solve fluid-structure interaction problem is very difficult, so numerical method to solve fluid-structure interaction is the current major research directions. Numerical method used there are usually two: one is uses the discrete finite element method for the fluid part and the structural elements, established the fluid and the solid coupling vibration equation; the other is uses the discrete finite element method for the fluid part, and the fluid is used boundary element method discrete, and then creates fluid-solid coupling vibration equation[2]..

This paper mainly discussed the fluid-solid coupling numerical method.

\section{The Numerical Algorithm}

The numerical solution of fluid-solid coupling algorithm can be divided into two types: loosely coupling and strongly coupling. Weakly coupling method and strongly coupling method were shown in Figure 1, the main difference between these two approaches is whether to "decouple" solving, and the decoupling is turned the original fluid-structure interaction from non-linear phenomena to a linear analysis.

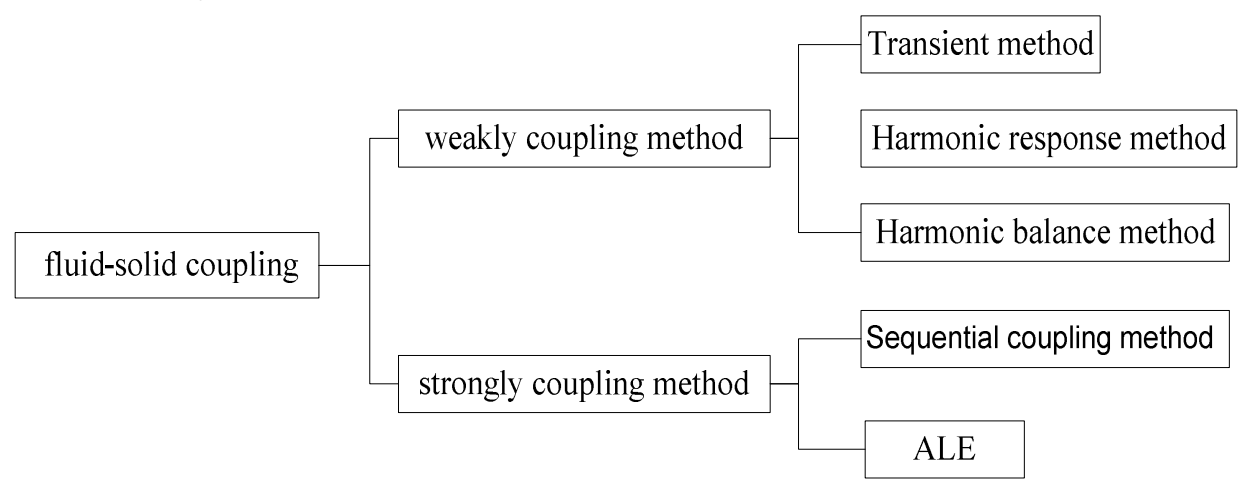

Fig. 1. The numerical solution of fluid-solid coupling algorithm

\section{Mathematical Method}

The Setup of Mathematical Model. Fluid-structure interaction problem can be decomposed three equations: the dynamic reaction equations of the structure, the dynamic reaction equations of the fluid, the boundary tolerate equation of fluid-solid interface [1].

1 The Mechanical Model of The Structure Dynamics (CSD)

The dynamic reaction of the structure is described by equation:

$$
\boldsymbol{M u}+\boldsymbol{C} \dot{\boldsymbol{u}}+\boldsymbol{K u}=\boldsymbol{F}(\boldsymbol{t}) .
$$

where $\boldsymbol{M}$ is mass matrix, $\boldsymbol{C}$ is damping matrix, $\boldsymbol{K}$ is rigidity matrix. $\boldsymbol{F}(\boldsymbol{t})$ represents the load added by the fluid, which includes surface pressure and shear stress. For the membrane structure, the shearing strength can not be neglected normally; but for the other structures, the shear strength put on by the fluid is very small and the structure is not sensitive to the shearing strength action. So the shear strength can be neglected, only the normal surface pressure is taken into consideration. The 
dynamic reaction of the structure is described by the structure's displacement $\boldsymbol{u}$ the speed $\dot{\boldsymbol{u}}$ the acceleration $\ddot{\boldsymbol{u}}$. Especially, the fluid damping is included in the right item of the equation (3), it is not in the damp matrix $\boldsymbol{C}$.

\section{Mathematical Model of the Fluid Dynamics (CFD)}

For the turbulence simulation, the RANS (Reynolds-Averaged Navier-Stockes) method is used in engineering calculations currently, the basic idea is to substitute the instantaneous values of density, velocities, enthalpy, scalar property, and so on with their mass-weighted mean and fluctuating quantities into the governing equations and taking the time average, the system of equations known as the Favre-Averaged Navier-Stockes equations can be established to approach the Reynolds equation for solution[8]. To solve the system of equations, the Reynolds and scalar stresses must be related to the mean quantities of the fluid field. The appropriate relationships of these stresses can be built by turbulent models, such as the standard $k-\varepsilon$ model, which is widely used in engineering, is adopted in this paper; this model is a semi-empirical model of the two equations of the turbulent kinetic energy $k$ and the diffusion rate $\varepsilon$, the control equations are as follows:

$$
\begin{aligned}
& \frac{\partial}{\partial t}(k \rho)+\frac{\partial}{\partial x_{j}}\left(\rho k u_{i}\right)=\frac{\partial}{\partial x_{j}}\left[\left(\mu+\frac{\mu_{t}}{\sigma_{k}}\right) \frac{\partial k}{\partial x_{j}}\right]+G_{k}-\rho \varepsilon \\
& \frac{\partial}{\partial t}(\rho \varepsilon)+\frac{\partial}{\partial x_{j}}\left(\rho \varepsilon u_{i}\right)=\frac{\partial}{\partial x_{j}}\left[\left(\mu+\frac{\mu_{t}}{\sigma_{\varepsilon}}\right) \frac{\partial \varepsilon}{\partial x_{j}}\right]+C_{1 \varepsilon} \frac{\varepsilon}{k} G_{k}-C_{2 \varepsilon} \rho \frac{\varepsilon^{2}}{k}
\end{aligned}
$$

where, $\rho$ represents the fluid's density, $\mu$ represents the fluid's dynamical viscosity coefficient, $\mu_{t}$ represents the turbulent viscosity coefficient, $G_{k}$ represents the turbulent kinetic energy created by the average velocity grads.

\section{Interpolation method of CFD-CSD}

Because CSD and CFD mesh disaccord, CSD mesh is sparseness then CFD mesh, so you must insert the CSD displacement of mesh node to CFD mesh node to make the data exchange.

The Interpolation method of commonly used: infinite plate spline method, finite plate spline method, multi-quadric-biharmonic method, thin spline method, inverse transform method with variable, non-uniform B-spline method.

Mathematical Simulation. The Fluid-solid coupling platform between the software CFX and ANSYS is MFX-ANSYS/CFX), which can exchange the calculation result of pressure in the flow field with the calculation result of displacement in the structure field. The MFX-ANSYS/CFX coupling platform is developed by the ANSYS Corporation to realize the coupling calculation between CFD and CSD.

This paper presented the FSI method of one-way coupling and two-way coupling of the engine blade. In engine, air considered as the fluid, and the blade considered as the structure. In this mathematical simulate model, the blade was modeled by the entity unit in ANSYS. The displacement restriction was the

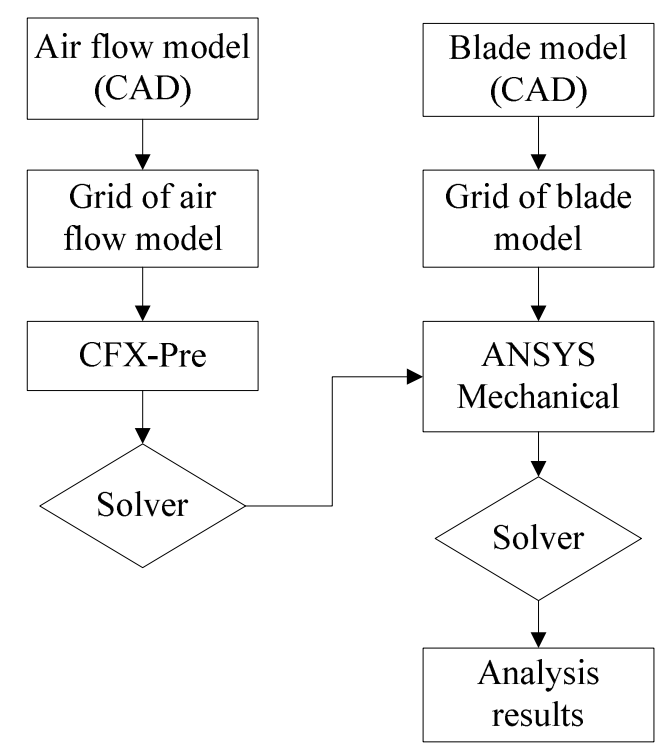

Fig. 2. One-Way Coupling Method 
displacement constraint between the blade and the hub; other surfaces were defined as fluid-solid interface. The revolving center was the axis of the rotor. The material of the blade was titanium alloy.

\section{One-Way Coupling Method}

1) Established simulate model (air flow model and blade model) in the CAD software, (such as the pro/E, Solidworks)

2) Divided grid with grid tools for model (such as Hypermesh, ICEM CFD).

3) Put the flow model mesh into CFX-Pre for pre-treatment, using ANSYS-CFX to get the temperature field and pressure field numerical calculation, using the $k-\varepsilon$ turbulence model. Set gas boundary conditions, the fluid domain displacement boundary conditions are mainly determined by the gas-solid coupling interface.

4) Solute the fluid analysis of the pre-treatment, generated results file.

5) Put the blade model grid file into ANSYS Mechanical, set the structure of the boundary conditions, adding a variety of loads, and put the CFX results file to the fluid-solid coupling of the interface.

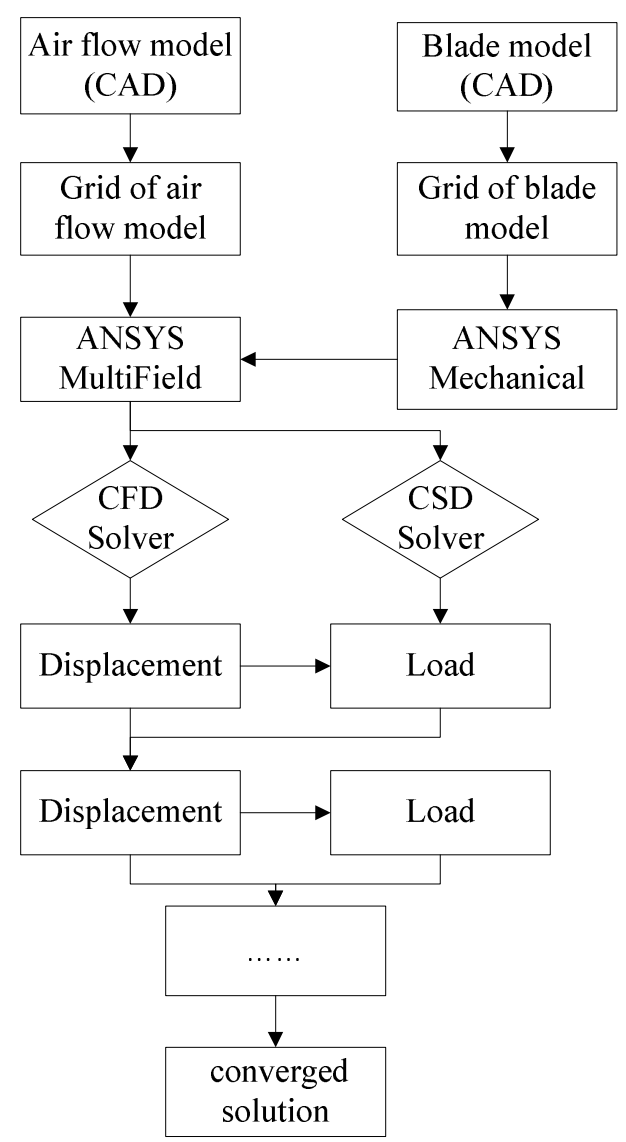

Fig. 3. Two-way Coupling Method

6) Analysis the results.

2 Two-way Coupling Method

1) Established simulate model (air flow model and blade model) in the CAD software, (such as the pro/E, Solidworks)

2) Divided grid with grid tools for model (such as Hypermesh, ICEM CFD).

3) Put the blade model grid file into ANSYS Mechanical, select the transient calculation, set the structure of the boundary conditions, adding a variety of loads, and export. Dat or. Inp file

4) Put the flow model mesh into CFX-Pre for pre-treatment, in the ANSYS-CFX Select ANSYS MultiField option and transient coupling analysis type, using the $k-\varepsilon$ turbulence model, abiding the heat transfer process conservation, momentum conservation and energy conservation law. Set gas boundary conditions, and get the pressure of the solid domain iterative calculations to the displacement of the fluid domain, then get the displacement of the fluid domain iterative calculations to the pressure of the solid domain [4].

5).Using the CFX-Slover tools to achieve the simultaneous calculation about CFD and CSD.

6) Analysis the results.

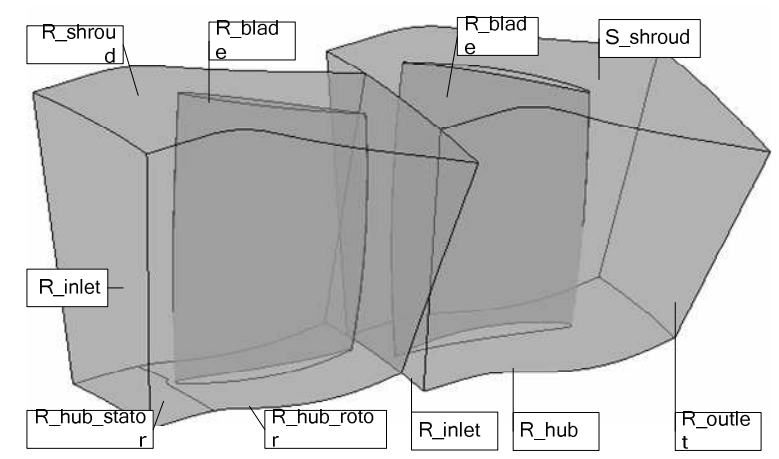

Fig. 4. A simplified model of fan-shaped flow channel area 


\section{Conclusions}

In engine, blades can be considered as the solids, and air which blades surrounded with can be considered as the fluid. And we study about blades deformation and displacement under the action of air loading in engine. Using computational structural dynamics (CSD) calculate the displacements of blades, and using computational fluidic dynamics (CFD) calculate the pressures of blades,
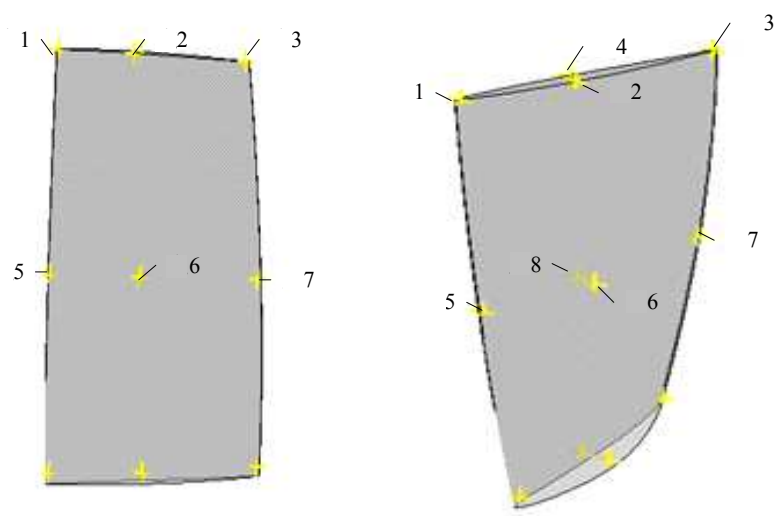

Fig. 5. The key points in the blades completing the fluid-structure interaction analysis in engine blades by iterating this two values(the displacements and the pressures) until the computational convergence solution is obtained. In this paper, the model of fluid-structure interaction and the simulate procession of the numerical analysis method were presented. Based on the analysis, the simulation result is qualitatively discussed referring to the factual conditions of the engine for validating the feasibility of analysis method.

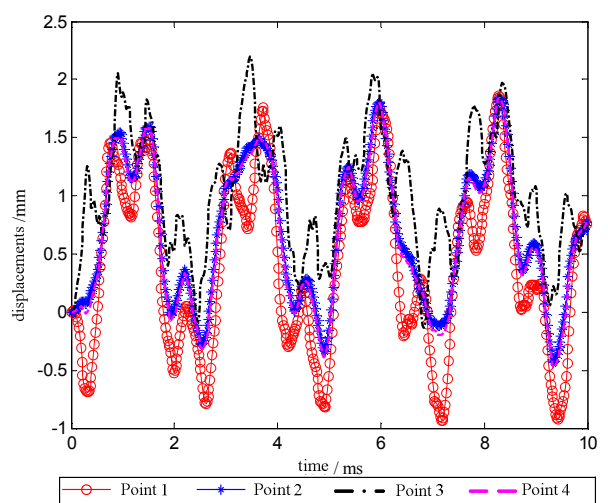

(a)The displacements of the point in tip blades

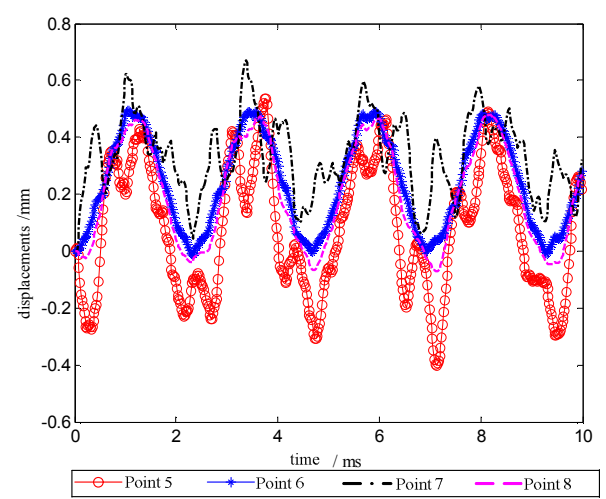

(c)The displacements of the point in middle blades

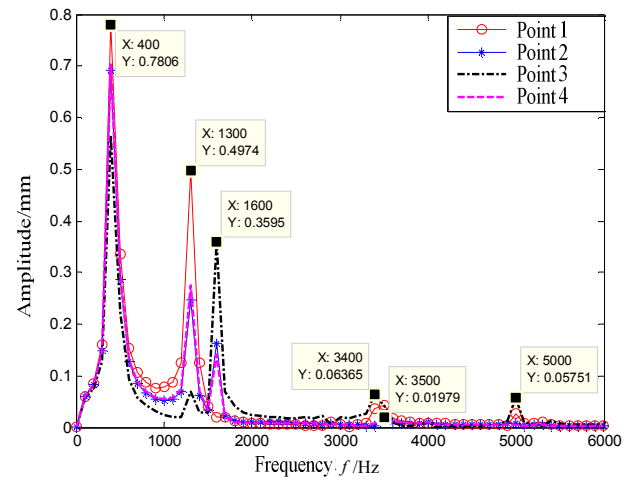

(b)The spectrum of the point in tip blades

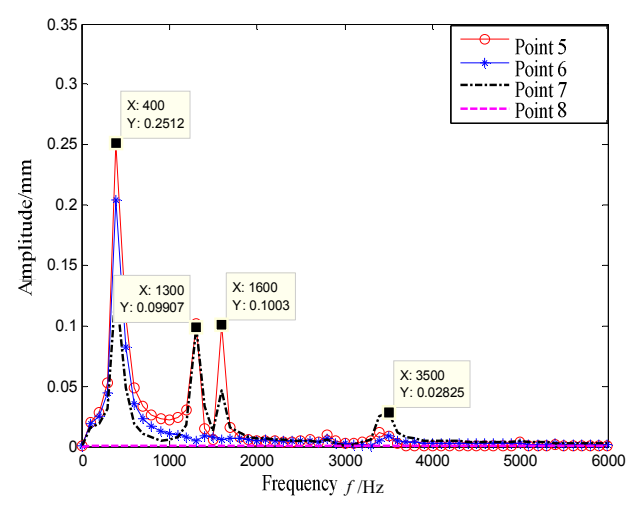

(b)The spectrum of the point in middle blades

Fig. 6. The displacements and the spectrum curves of the key point in blades with the speed of $785 \mathrm{rad} / \mathrm{s}$ 


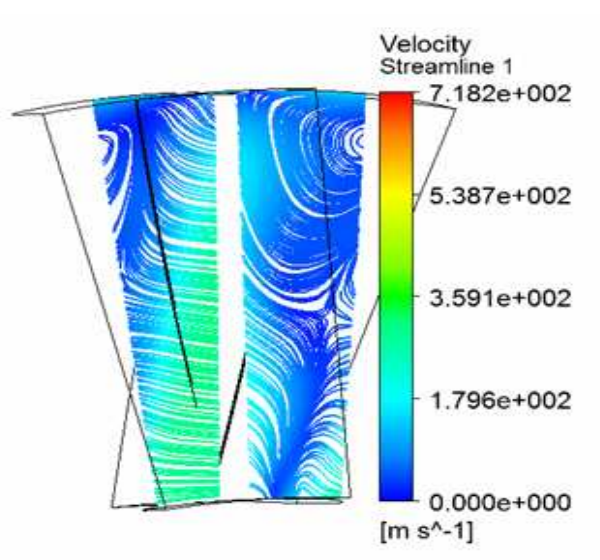

(a) The flow chart of blades sides

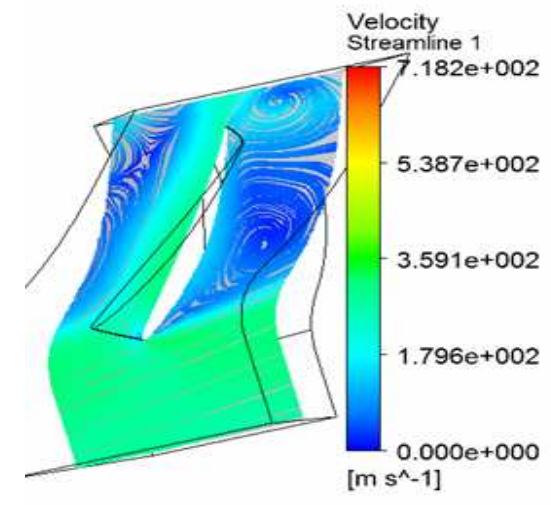

(b) The flow chart of blades cross section

Fig. 7. The vortex within the fluid flow channel as the role of the flutter with the speed of $785 \mathrm{rad} / \mathrm{s}$

According to the method, we have setup a single-passageway model (including a the rotor blade and a stator blade) were shown in Figure 4. And analyzed with one-way coupling method and two-way coupling method, from the analysis we can get the pressure spectrum and displacement spectrum analysis.Get 12 points from the blade, shown in Figure 5. Calculated the X direction of displacement of 12 key points in time, and plotted the displacement curves and spectrum curves using MATLAB software were shown in Figure 6 (a) - (d). We can find the blade displacement of the tip is greater than other locations, especially near the outlet location, which may be caused by the movement interference. As the role of the flutter, the vortex within the fluid flow channel may be generated, and observed vortex around with blades in Figure 7, and similar to the previous forecast. And we can have a better understanding of the blade work environment, and optimize the design of the structure of blades, as a data support for optimization of design.

\section{Acknowledgement}

This work was financially supported by Natural Science Foundation of China (51005043) and the Fundamental Research Funds for the Central Universities (N100303004).

\section{References}

[1] Zhang X, Wang Y R, Zhang X W, Pei W. The method of Fluid-structure Interaction Based on Multi-level Grid Technology [J],Ship engineering, 2009,31(1):64-74

[2] Cao L. Analysis of Fluid-Solid Coupling Vibration of Francis Turbine [D], Kunming University of Science and Technology, 2007

[3] Zhang X W, Wang Y R, Zhang X, Pei W. Numerical Method for Fluid-Structure Interaction of Eddy Mechanical blade [J], Journal of Aerospace Power, 2009,24(7):1622 1626

[4] Zhang D Y, Ma Y H, Hong J, Chen L L. The Method for Dynamic Response Analysis of blades under Air Flow excitation [J], Journal of Aerospace Power, 2009,24(7):1523 1529 